\title{
Software-based simulation for preprocedural assessment of Tubridge flow diverter sizing: a validation study
}

Jia-nan Li

Changhai Hospital https://orcid.org/0000-0002-9776-2129

Laura Obradó

Galgo Medical S.L

Ignacio Larrabide

Galgo Medical S.L

Héctor Fernández

Galgo Medical S.L

Nan LV

Changhai Hospital

Qiang Li

Changhai Hospital

Peng-fei Yang

Changhai Hospital

Qing-hai Huang

Changhai Hospital

Yi Xu

Changhai Hospital

Bo Hong

Changhai Hospital

Jian-min Liu

Changhai Hospital

Yi-bin Fang ( $\nabla$ fangyibin@163.com )

Research

Keywords: Tubridge flow diverter; soft simulation; aneurysm

Posted Date: December 16th, 2019

DOI: https://doi.org/10.21203/rs.2.18768/v1 
License: (c) (i) This work is licensed under a Creative Commons Attribution 4.0 International License. Read Full License 


\section{Abstract}

Background: The purpose of this single-center retrospective review was to validate the use of a softwarebased simulation of Tubridge flow diverter (FD) in the treatment of intracranial aneurysms. In a singlecenter cohort of 17 patients undergoing aneurysm treatment with the Tubridge flow divert, we analyzed their pre- and post-procedural angiographic studies to compare the manufacturer-given nominal length $(\mathrm{NL})$, software calculated simulated length $(\mathrm{SL})$, and the actual measured length $(\mathrm{ML})$ of the flow divert using software.

Results: Data for the 3 lengths of all 17 patients treated with Tubridge flow diverts were collected and analyzed in this study. Error discrepancy was calculated by mean squared error (ML to NL 10.64; SL to NL $9.95 p>0.05$ ), mean absolute error ( $M L$ to NL 2.64; SL to NL $2.60 p>0.05$ ), and mean error (NL to $M L 1.26$; SL to ML $2.59 \mathrm{p}>0.05)$.

Conclusions: The SL was usually greater than the NL given by the manufacturer, indicating significant change in length in most cases. The residual comparing the ML to the NL was significant, as was when comparing the SL to the NL. The assessment of the Tubridge flow diverter using software simulation is safe and effective and the accurate calculating of FD length contributed to the right-sized FD for optimal placement in intracranial vasculature.

\section{Background}

Flow-diversion concept was originally formulated for aneurysm treatment in the 1990s.[1, 2] With the development of material technology, several new flow diverter (FD) devices, such as the Pipeline Embolization Device (Covidien, Irvine, California), the Silk flow diverter (Balt Extrusion, Montmorency, France), the Flow-Redirection Endoluminal Device (FRED; MicroVention, Tustin, California), and the Surpass stent (Stryker Neurovascular, Kalamazoo, Michigan) have been available, and animal experiments have been performed to prove their efficacy and safety.[3-5] The Tubridge FD, developed by MicroPort NeuroTech, Shanghai, China, is a new type of flow diversion device on the basis of our previous hemodynamic studies of intracranial blood flow, aimed at treating complex aneurysms, such as large and giant aneurysms, and providing more treatment options for neurointerventionists and neurosurgeons.[6] The size of FD is usually determined by the diameter of the distal and proximal parent artery, which might change along the vasculature due to its self-expanding character.[7] There is no accurate method to predict the change in length and exact foreshortening before the deployment of the flow diverter.

Advances computer based modelling tools for flow diverter size or braided self-expending stent have been reported before, and have not been used before for Tubridge FD sizing.[8, 9] We present quantitative data of Tubridge FD sizing using software and the actual FD size after deployment.

\section{Results}

Baseline, treatment characters, perioperative complications and outcome 
Seventeen patients from our center were included consecutively, including 9 females and 8 males with mean age 55.6 years (range 6 to 78 years). Total 19 aneurysms were demonstrated by digital subtraction angiography (DSA) in these patients, 15 cases with single aneurysm, and 2 cases with multiple intracranial aneurysms. Eight of the aneurysms were large. Aneurysm locations were as follows: internal carotid artery (ICA) communicating segment $(n=3)$, ICA ophthalmic segment $(n=6)$, ICA paraclinoid segment $(n=2)$, ICA cavernous segment $(n=4)$, middle cerebral artery $(n=1)$, anterior cerebral artery $(n=1)$ and vertebral artery (VA) intradural segment $(n=2)$. The original mean maximum diameter was $10.23 \mathrm{~mm}$ (range: 3-20.9mm).

Eighteen Tubridge FDs were successfully deployed in all 17 patients (Table 1). A single Tubridge FD was used in 7 cases, Tubridge FD+ coiling in 9 cases (52.9\%), and multiple Tubridge FD in one case (5.9\%). In the case in which multiple Tubridge FD constructs were used, analysis was limited to the first implanted device. Periprocedural ischemia was detected in one case (5.9\%) in the same territory where the Tubridge FD had been deployed. Six-month imaging follow-up was available for $11 / 17$ patients $(64.7 \%)$ and revealed residual aneurysm in one patient $(9.1 \%)$.

\begin{tabular}{ccc}
\multicolumn{3}{c}{ Table.1 Baseline, FD size and outcome } \\
\hline No. & FD size & Follow up outcome \\
\hline 1 & $3.5 \times 30$ & Complete occlusion \\
\hline 2 & $4.0 \times 20$ & Complete occlusion \\
\hline 3 & $5.0 \times 35$ & Complete occlusion \\
\hline 4 & $5.0 \times 45$ & Complete occlusion \\
\hline 5 & $4.0 \times 45$ & Complete occlusion \\
\hline 6 & $4.0 \times 25$ & NA \\
\hline 7 & $4.5 \times 35$ & NA \\
\hline 8 & $4.5 \times 20$ & Complete occlusion \\
\hline 9 & $4.0 \times 35$ & Complete occlusion \\
\hline 10 & $4.0 \times 15$ & NA \\
\hline 11 & $4.0 \times 30$ & Complete occlusion \\
\hline 12 & $4.0 \times 35$ & Complete occlusion \\
\hline 13 & $4.0 \times 45$ & residual aneurysm \\
\hline 14 & $5.0 \times 30$ & NA \\
\hline 15 & $4.5 \times 25$ & NA \\
\hline 16 & $3.0 \times 15$ & NA \\
\hline 17 & $2.5 \times 20$ & Complete occlusion \\
\hline
\end{tabular}

\section{Tubridge dimensions}

Figure 1 shows the distribution of length changes observed between nominal and measured lengths in the cases studied. The frequency is computed for the relative device length change compared to the nominal (measured length - nominal length)/ nominal length $\times 100 \%$ ). Changes of up to $50 \%$ with respect 
to nominal length in stent length occurred, with a mean change of $5 \%$. It was also observed that changes could be negative, indicating that the final length could be smaller than the nominal length.

The error discrepancy between these measurements was calculated using three standard measures of error (Table 2). The mean squared error (MSE) between the measured and nominal values and between the simulated and nominal values were $10.64 \mathrm{~mm}$ and $9.95 \mathrm{~mm}$ respectively. The mean absolute error (MAE) use the same scale as the data being measured, and it ignores whether it is an under- or overprediction. The MAE between measured and nominal values and between simulated and nominal values were $2.64 \mathrm{~mm}$ and $2.60 \mathrm{~mm}$ respectively. These values were charted on a scatterplot, which shows a positive correlation between measured and nominal lengths (rho $=0.952, p<0.01$, Figure 2), as well as between simulated and nominal values (rho=0.991, $p<0.01$, Figure 2). Mean error (ME) was the third measure, which represents the average of errors in the complete set, taking into account any under- or over-prediction. ME between measured and nominal lengths and between simulated and nominal lengths were $1.26 \mathrm{~mm}$ and $2.59 \mathrm{~mm}$ respectively. It means both the measured length and stimulated length were greater than the nominal length as designed. The Wilcoxon signed-rank paired test showed that the nominal and simulated lengths were significantly different $(p<0.01)$, the nominal was $1.26 \mathrm{~mm}$ shorter than the measured length. The simulated length was as accurate as the measured length of the implanted device which is supported by the error measures. These values were charted on a Bland-Altman plot show the mean difference in length between the measured and simulated lengths, the mean difference is $-1.3 \mathrm{~mm}$ (median-1.78mm). (Figure 3).

Table2. Discrepancy in the degree of error between simulated and measured lengths: the nominal length

\begin{tabular}{|l|l|l|l}
\hline Length & MSE & MAE & ME \\
\hline measured to nominal & 10.64 & 2.64 & 1.26 \\
\hline simulated to nominal & 9.95 & 2.60 & 2.59 \\
\hline
\end{tabular}

Pearson's correlation analysis showed a rho of $0.952(p<0.001)$ when comparing measured to nominal lengths, and a rho of $0.991(p<0.001)$ when comparing simulated to nominal lengths. A dependent 2group Wilcoxon signed-rank test (paired) was performed for the residuals with a $p=0.055$ was found. This means that the mean of the residuals can be assumed to be equal to 0 . Furthermore, the residual comparing the measured to the nominal length was found to be significant, same as the residual comparing the simulated to the nominal length.

\section{Discussion}

Flow diverters were designed to treat complex aneurysms that were difficult to be handled by surgical clipping or conventional endovascular treatments, and have been increasingly used in more than 50 countries.[7] These devices were believed to improve long-term effectiveness, due to the capability to alter intravascular hemodynamics.[7] Preliminary evidence has demonstrated their safety and efficacy for treating complex aneurysms.[6] 
The Tubridge FD is a stent-like vessel-reconstruction devise designed with a high metal coverage rate and low porosity and was approved by the Ethics Committee of our institution and by the Chinese Food and Drug Administration. This FD is available in various diameters and lengths, which were designed with a pore size of $0.040-0.050 \mathrm{~mm}^{2}$ at the nominal diameter to provide high metal coverage at the neck of the aneurysm after full opening. The large size Tubridge FD is a braid of 62 nickel-titanium microfilaments and 2 platinum-iridium radio-opaque microfilaments; the small Tubridge is composed of 46 nitinol and 2 platinum-iridium microfilaments.[10] Result of the PARAT study, a Chinese multicenter RCT, published recently showed that there was a significantly higher obliteration rate of aneurysms treated with the Tubridge FD when compared to stent assisted coiling in large or giant intracranial aneurysms. The complication rates were comparable in two groups. The authors mentioned the complication rate was even lower in the primary investigational site, where the leading physicians already had gained considerable experience with the Tubridge FD. However, the selection and deployment of Tubridge FD is still technically challenging.[11] Therefore, it’s useful to optimize device sizing and device positioning through preoperative simulation. Computer-based simulation modeling tools like ANKYRAS provide the possibility to simulate and anticipate Tubridge FD behavior of variable dimensions easily and efficiently. This technique aims to make device selection standardized and to help the physicians in the decisionmaking during the procedures.

The 3D angiographic image before stent deployment was used to calculate the simulated length using software and the shape of aneurysm and variable diameter around the curves were taken into account. Compared with the nominal length provided by the company, the simulated length was found to be as accurate as the measured length. The simulation could help the neurointerventionist choose the adequate size of the FD, especially for physicians without extensive experience. Similar studies have been done with other flow diverters and braided stents, such as the Pipeline, Derivo and Lvis, and have shown that simulation can be useful during stent deployment.[12] Ospel et al supposed that simulated length of Pipeline calculated by software was shorter than the manually determined presumed optimal devices.[13] In our study a longer devices was calculated by software than physicians' selection. This may result from the material and structural differences between the Tubridge and the Pipeline FD. Deployment of the FD requires a combination"push" and "pull" technique to ensure full opening of the device and to increase the apposition along the device.[6] The Tubridge was made of a nickel-titanium alloy known as nitinol, and Pipeline FD was made of a cobalt-chromium alloy. Because of the softer characteristic of nickel-titanium alloy, it is more susceptible to be pushed and pulled, which exhibits better shape-memory and superelasticity than cobalt-chromium alloy. This phenomenon is similar to that of Lvis stent made of nickel-titanium alloy. The Tubridge FD could offer multiple structural improvements over the Pipeline FD. The use of more braided microfilaments for the large-size Tubridge decreases the shorting rate after full opening and offers more appropriate pore attenuation compared with the 48-wire design of the Pipeline flow diverters. Pipeline is more difficult to be pushed, so manually determined length would be longer than simulated length.

The morphology of flow diversion device could only be acquired after implantation, and preoperative simulation calculated by the software would provide visual reference to the neurointerventionist. Although 
simulated length calculated by the software was significantly greater than nominal length in our study, the residual between the measured and the nominal length was found to be the same as the residual between the simulated and the nominal length. Real-time stimulation with ANKYRAS could provide great value in clinical decision making. The device deformation, porosity, and degree of wall apposition is also provided. Different device diameters and lengths could be virtually tested in real-time.

Software-based planning tools that visualize the length of flow diverter have been described.[14-16] The first generation of software simulation included the direct placement method, where a uniform stent tube was simply fitted into a patent vessel.[17, 18$]$ However, the first generation of software simulation deviates from actual result greatly, because the length of braided stent after implantation is greatly affected by the diameter of blood vessel and poor stent-wall apposition. Other software-based simulation methods were described, such as finite element method, unstructured embedded grids method and fast virtual stenting method.[15, 19] The limitation of previous software solutions has ignored the difference between the intracranial vasculature and vivo models, such as a lack of information regarding wall apposition and the inability to test, in real-time, multiple device dimensions and positions.[19] The assessment and measurement of vascular structure in real time in a real-world setting is the greatest challenge for software simulation, particularly for complex aneurysms.

This is the first clinical study to validate the role of software-assisted simulation in Tubridge FD treatment of intracranial aneurysms. Our results represent an important step toward translation of this novel flow diverter into routine clinical practice and provide effective FD sizing method for the neurointerventionist.

\section{Conclusion}

The assessment of the Tubridge FD using software simulation is safe and effective and the accurate calculating of FD length contributed to the right-sized FD for optimal placement in intracranial vasculature.

\section{Methods}

Angiographic data of 17 consecutive patients, whom undergoing aneurysm treatment with Tubridge FD between May 2018 and September 2018 in our center, were retrospectively collected. The retrospective study was approved by institutional review board of our hospital, and the informed consents were waived.

\section{Baseline characteristics}

Baseline characteristics included patient gender and age, as well as aneurysm location and maximum diameter. We recorded the additional use of embolic material such as coils and whether a single Tubridge or multi-Tubridge construct was used.

\section{Perioperative complications and outcome}


Perioperative complications, such as Tubridge deployment failure, occlusion of covered branches, and occurrence of hemorrhage or ischemic stroke, were recorded. Radiologic outcome, including the presence of residual aneurysm perfusion, was recorded for all patients with 6 months imaging follow-up.

\section{Stent length measurement}

Three important variables were documented: 1) nominal length of the Tubridge, which was stipulated by the manufacturer; 2) simulated length, which was calculated by the software; and 3) measured length which was measured by a neurointerventionist after deployment. The length of the implanted Tubridge inside the artery was measured from 2D angiographic sequences after operation. The 3D model obtained from the 3DRA images performed before operation was used to generate a centerline. The 3D model was manually registered to the same point of view as the 2D image were acquired. The contrast phase obtained from 2D sequence was used to determine the anatomical location and orientation for a precise alignment between 3D model and 2D image sequence. Finally, the distal and proximal markers of the device were identified on the 2D image and projected on the centerline. The length of the Tubridge FD was calculated as the distance between the distal end and proximal end along the centerline. Biplane acquisitions were available in all cases to choose more clear visibility.

\section{Simulation of FD length}

Tubridge FD length was simulated using the braided device foreshortening (BDF) algorithm. A 3D model of the vessel is used to extract the centerline and characterize the local vessel morphology. The computational models used to simulate each device are based on numbers of wires of the stent and the length at two different diameters, which was available from device manufacturer specifications. The obtained model was used to parameterize the simulation following the procedure described by Fernandez[20].

Local morphological descriptors were used to assess length change at each region of the vessel. The distal end position of the simulated FD was matched to the position observed on the 2D angiographic sequences and the proximal one was obtained by running the BDF algorithm. The final length of the FD (simulated length) is the distance between the distal end and the proximal end of the simulated FD along the centerline.

\section{Error assessment between measurements}

The simulation was assessed using an error measure. The error between both the measured length and the simulated length was compared to the nominal length stated by the manufacturer using the following criteria:

MSE $\left[\mathrm{mm}^{2}\right](L, N)=$ mean $\left((L-N)^{2}\right)$ The mean squared error (MSE) that accounts for the squared differences between nominal length and approximated stent length. 
MAE $[\mathrm{mm}](\mathrm{L}, \mathrm{N})=$ mean $|\mathrm{L}-\mathrm{N}|$ The mean absolute error (MAE) that quantifies the absolute differences between nominal length and approximated stent length.

$M E[m m](L, N)=$ mean $(L-N)$ The mean error $(M E)$ represents the mean difference between nominal length and approximated stent lengths.

In the above, $L$ is either the measured (M) length or the simulated (S) length, and $N$ is the nominal length stated by the manufacturer.

\section{Statistical Analysis}

Differences in baseline characteristics were evaluated with a Student $t$ test, Fisher exact test, and Wilcoxon signed rank test, as applicable. The Statistical Package for the Social Sciences (SPSS, Chicago, USA) was used for statistical analysis, and a $p$-value $<0.05$ was considered statistically significant. Bland-Altman plot was used to evaluate the consistency of simulated or nominal and measured lengths.

\section{Abbreviations}

NL: nominal length; SL: simulated length; ML: measured length; FD: flow diverter; DSA: digital subtraction angiography; ICA: internal carotid artery; VA: vertebral artery; BDF: braided device foreshortening; MSE: mean squared error; MAE: mean absolute error; ME: mean error

\section{Declarations}

\section{Acknowledgements}

Not applicable.

\section{Authors' contributions}

YBF conceived the study and developed the methods; JNL and NL collected data; LO, HF, and JNL sorted and analyzed the data. JNL drafted the manuscript; JNL prepared the figures; LL, QL, PFY, QHH, YX, BH, and JML provided critical review of the manuscript. All authors have reviewed the final version of the manuscript for publication. All authors read and approved the final manuscript.

\section{Funding Statement}

This work was supported by the National Key r\&D program of China (2016YFC1300700), National Natural Science Foundation of China (No 81301004), and project on research and application of effective intervention techniques for high risk population of stroke from the National Health and Family Planning Commission in China (GN-2016r0012).

\section{Availability of data and materials}


Not applicable.

\section{Ethics approval and consent to participate}

Not applicable.

\section{Consent for publication}

All authors have approved the manuscript and agreed with submission and publication. The manuscript has not previously been published elsewhere and is not under consideration by any other journals.

\section{Competing interests}

The authors declare that they have no competing interests.

\section{References}

1. Turjman F, Acevedo G, Moll T, Duquesnel J, Eloy R, Sindou M. Treatment of experimental carotid aneurysms by endoprosthesis implantation: preliminary report. Neurol Res 1993, 15(3):181-184.

2. Geremia G, Haklin M, Brennecke L. Embolization of experimentally created aneurysms with intravascular stent devices. AJNR Am J Neuroradiol 1994, 15(7):1223-1231.

3. Kim BM, Kim DJ, Kim DI. A New Flow-Diverter (the FloWise): In-Vivo Evaluation in an ElastaseInduced Rabbit Aneurysm Model. Korean J Radiol 2016, 17(1):151-158.

4. Huang QH, Yang PF, Zhang X, Shi Y, Shao XM, Liu JM. Effects of flow diverter with low porosity on cerebral aneurysms: a numerical stimulative study. Zhonghua yi xue za zhi 2010, 90(15):1024-1027.

5. Simgen A, Ley D, Roth C, Yilmaz U, Korner H, Muhl-Benninghaus R, Kim YJ, Scheller B, Reith W. Evaluation of a newly designed flow diverter for the treatment of intracranial aneurysms in an elastase-induced aneurysm model, in New Zealand white rabbits. Neuroradiology 2014, 56(2):129137.

6. Zhou Y, Yang PF, Fang YB, Xu Y, Hong B, Zhao WY, Li Q, Zhao R, Huang QH, Liu JM. A novel flowdiverting device (Tubridge) for the treatment of 28 large or giant intracranial aneurysms: a singlecenter experience. AJNR Am J Neuroradiol 2014, 35(12):2326-2333.

7. Sadasivan C, Cesar L, Seong J, Wakhloo AK, Lieber BB. Treatment of rabbit elastase-induced aneurysm models by flow diverters: development of quantifiable indexes of device performance using digital subtraction angiography. IEEE Trans Med Imaging 2009, 28(7):1117-1125.

8. Xiang J, Damiano RJ, Lin N, Snyder KV, Siddiqui AH, Levy El, Meng H. High-fidelity virtual stenting: modeling of flow diverter deployment for hemodynamic characterization of complex intracranial aneurysms. J Neurosurg 2015, 123(4):832-840.

9. Joshi KC, Larrabide I, Saied A, Elsaid N, Fernandez H, Lopes DK. Software-based simulation for preprocedural assessment of braided stent sizing: a validation study. J Neurosurg 2018:1-7. 
10. De Vries J, Boogaarts J, Van Norden A, Wakhloo AK. New generation of Flow Diverter (surpass) for unruptured intracranial aneurysms: a prospective single-center study in 37 patients. Stroke 2013, 44(6):1567-1577.

11. Zhou Y, Yang PF, Fang YB, Xu Y, Hong B, Zhao WY, Li Q, Zhao R, Huang QH, Liu JM. Parent artery reconstruction for large or giant cerebral aneurysms using a Tubridge flow diverter (PARAT): study protocol for a multicenter, randomized, controlled clinical trial. BMC Neurol 2014, 14:97.

12. Suzuki T, Takao H, Fujimura S, Dahmani C, Ishibashi T, Mamori H, Fukushima N, Yamamoto M, Murayama Y. Selection of helical braided flow diverter stents based on hemodynamic performance and mechanical properties. J Neurointerv Surg 2017, 9(10):999-1005.

13. Ospel JM, Gascou G. Comparison of Pipeline Embolization Device Sizing Based on Conventional 2D Measurements and Virtual Simulation Using the Sim\&Size Software: An Agreement Study. AJNR Am J Neuroradiol. 2019, 40(3):524-530.

14. Kim JH, Kang TJ, Yu WR. Mechanical modeling of self-expandable stent fabricated using braiding technology. J Biomech 2008, 41(15):3202-3212.

15. Bernardini A, Larrabide I, Petrini L, Pennati G, Flore E, Kim M, Frangi AF. Deployment of selfexpandable stents in aneurysmatic cerebral vessels: comparison of different computational approaches for interventional planning. Comput Methods Biomech Biomed Engin 2012, 15(3):303311.

16. Bouillot P, Brina O, Yilmaz H, Farhat M, Erceg G, Lovblad KO, Vargas MI, Kulcsar Z, Pereira VM. Virtualversus-Real Implantation of Flow Diverters: Clinical Potential and Influence of Vascular Geometry. AJNR Am J Neuroradiol 2016, 37(11):2079-2086.

17. Fu W, Gu Z, Meng X, Chu B, Qiao A. Numerical simulation of hemodynamics in stented internal carotid aneurysm based on patient-specific model. J Biomech 2010, 43(7):1337-1342.

18. Kim M, Taulbee DB, Tremmel M, Meng H. Comparison of two stents in modifying cerebral aneurysm hemodynamics. Ann Biomed Eng 2008, 36(5):726-741.

19. Appanaboyina S, Mut F, Löhner R, Putman C, Cebral J, Cma J, Stenting. Simulation of intracranial aneurysm stenting: Techniques and challenges. Computer Methods in Applied Mechanics \& Engineering 2009, 198(45):3567-3582.

20. Fernandez H, Macho JM, Blasco J, Roman LS, Mailaender W, Serra L, Larrabide I. Computation of the change in length of a braided device when deployed in realistic vessel models. Int $\mathrm{J}$ Comput Assist Radiol Surg 2015, 10(10):1659-1665.

\section{Figures}




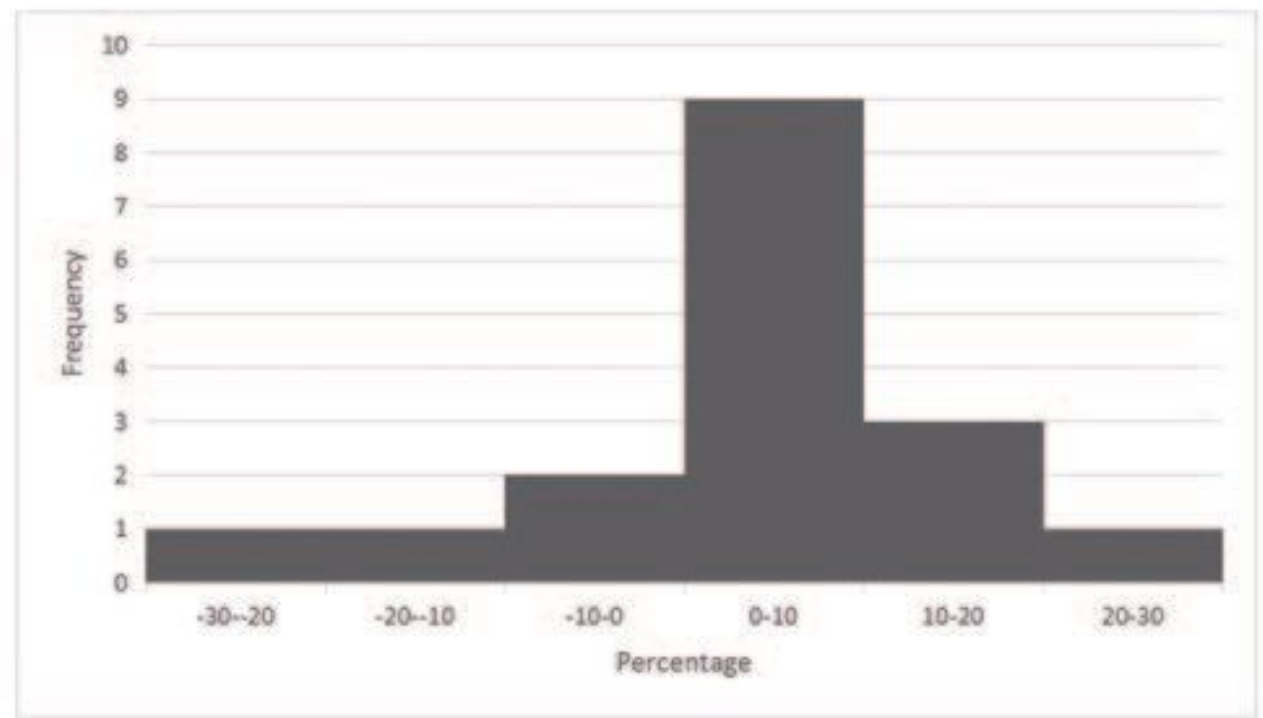

\section{Figure 1}

The distribution of length changes observed between nominal and measured lengths in the cases studied.
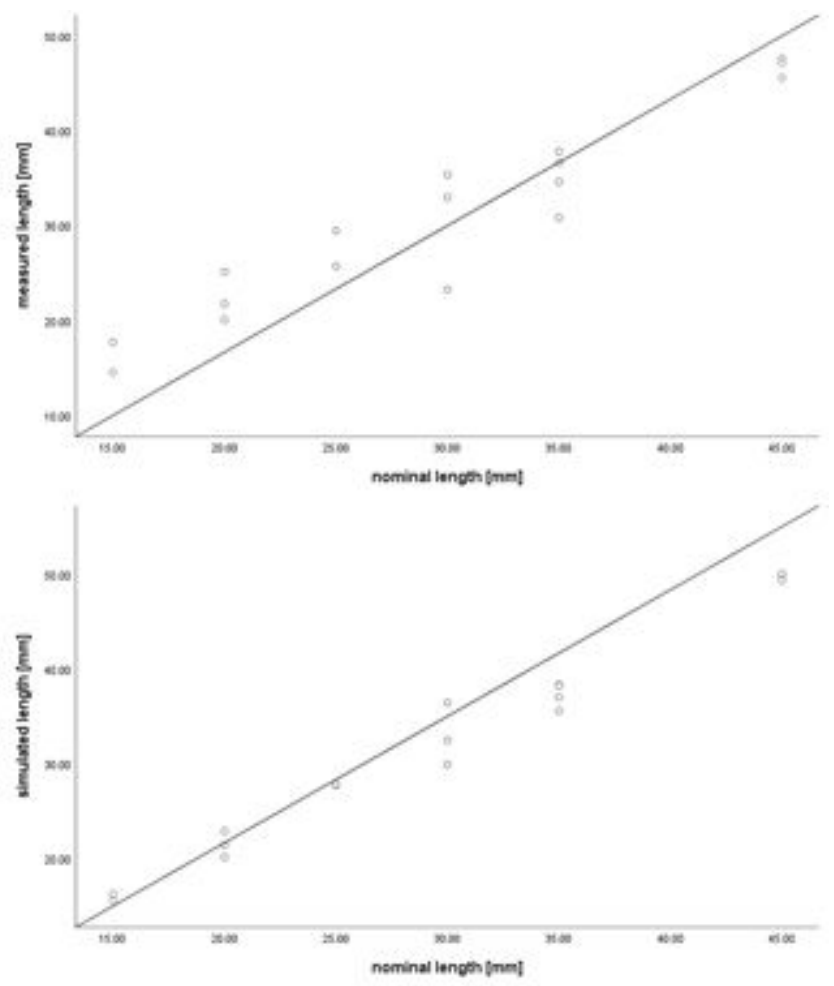

Figure 2

These values were charted on a scatterplot, which shows a positive correlation between measured and nominal lengths ( $r h o=0.952, p<0.01$ ), as well as between simulated and nominal values (rho $=0.991$, $\mathrm{p}<0.01)$. 


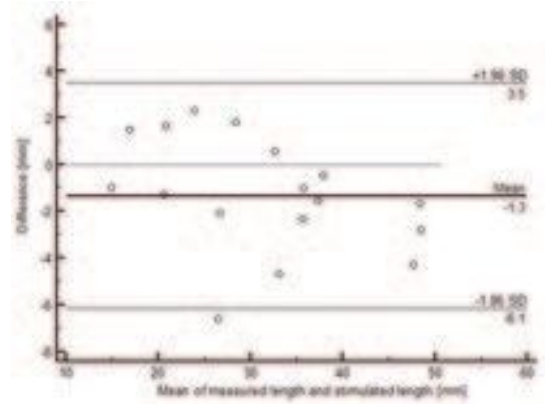

\section{Figure 3}

Bland-Altman plot shows the mean difference in length between the measured and simulated lengths, the mean difference is $-1.3 \mathrm{~mm}$ (median-1.78mm). 